\title{
THE SECOND ITERATE OF A MAP WITH DENSE ORBIT
}

\author{
PAUL S. BOURDON
}

(Communicated by James E. West)

\begin{abstract}
Suppose that $X$ is a Hausdorff topological space having no isolated points and that $f: X \rightarrow X$ is continuous. We show that if the orbit of a point $x \in X$ under $f$ is dense in $X$ while the orbit of $x$ under $f \circ f$ is not, then the space $X$ decomposes into three sets relative to which the dynamics of $f$ are easy to describe. This decomposition has the following consequence: suppose that $x$ has dense orbit under $f$ and that the closure of the set of points of $X$ having odd period under $f$ has nonempty interior; then $x$ has dense orbit under $f \circ f$.
\end{abstract}

\section{INTRODUCTION}

Suppose that $T$ is a linear function on a complex Banach space $B$ and that the orbit of $b \in B$ under $T$ is dense in $B$; then for each positive integer $n$, the orbit of $b$ under $T^{n}$ is also dense. This remarkable result was recently obtained by S. I. Ansari ([1, Theorem 1]). Her proof makes use of the fact that once $T$ has a single vector $b$ with dense orbit, it has a dense connected set of vectors having dense orbit.

Inspired by Ansari's work, we move in this paper to a topological-space setting in which a continuous map $f$ acts on a Hausdorff space $X$ having no isolated points (no linearity assumptions on space or map). We examine what must happen if a point $x$ has dense orbit under $f$ while failing to have dense orbit under $f \circ f$. Our main result, the Separation Theorem, yields Ansari's theorem in the $n=2$ case; more important, it leads to a decomposition of $X$ into three sets relative to which the dynamics of $f$ are easy to describe. This decomposition, in turn, yields the following: suppose that $f$ acts chaotically on $X$ and that the closure of the set of points of $X$ having odd period under $f$ has nonempty interior; then $f \circ f$ acts chaotically on $X$.

\section{The Separation Theorem}

In this section and the one that follows, $X$ denotes a Hausdorff topological space having no isolated points, and $f$ denotes a continuous map from $X$ to $X$. We use $f^{[n]}$ to represent $f$ composed with itself $n$ times:

$$
f^{[n]}=f \circ f \circ f \circ \cdots \circ f, n \text { times }
$$

(take $f^{[0]}$ to be the identity map). For a subset $S$ of $X$,

$$
f^{-[n]}(S):=\left\{w \in X: f^{[n]}(w) \in S\right\} .
$$

Received by the editors June 1, 1994.

1991 Mathematics Subject Classification. Primary 54H20; Secondary 47A15, 58F13.

(C)1996 American Mathematical Society 
For $x \in X$, the orbit of $x$ under $f$, denoted $\operatorname{Orb}(f, x)$, is given by

$$
\operatorname{Orb}(f, x)=\left\{f^{[n]}(x): n=0,1,2, \ldots\right\} .
$$

Let $\mathcal{D}$ be the set of elements of $X$ that have dense orbit under $f$. We show that if $\operatorname{Orb}(f, x)$ is dense in $X$ while $\operatorname{Orb}\left(f^{[2]}, x\right)$ is not, then there exists a separation of $\mathcal{D}$. Before proving this separation theorem, we present two simple lemmas.

Lemma 2.1. Suppose that $\mathcal{D}$ is not empty. Then $\mathcal{D}$ is a dense subset of $X$ fully invariant under $f$ :

$$
f(\mathcal{D}) \subset \mathcal{D} \quad \text { and } \quad f^{-[1]}(\mathcal{D}) \subset \mathcal{D} .
$$

Proof. Because $X$ is a Hausdorff space having no isolated points, removing a finite set from a dense set leaves a dense set. Let $x$ be in $\mathcal{D}$. Then $\operatorname{Orb}(f, f(x))$ is dense in $X$ since it is the dense set $\operatorname{Orb}(f, x)$ minus the singleton set $\{x\}$. Hence $f(x) \in \mathcal{D}$, and $\mathcal{D}$ is invariant under $f$. Observe that as a consequence of this invariance, given $x$ is in $\mathcal{D}, \operatorname{Orb}(f, x)$ must be contained in $\mathcal{D}$; however, $\operatorname{Orb}(f, x)$ is dense in $X$ and thus $\mathcal{D}$ is dense in $X$ as well.

That $f^{-[1]}(\mathcal{D}) \subset \mathcal{D}$ is clear because the orbit of a given $y \in f^{-[1]}(\mathcal{D})$ contains $\operatorname{Orb}(f, f(y))$, which is dense in $X$.

Lemma 2.2. Suppose that $x \in X$, that $h: X \rightarrow X$ is continuous, and that $G$ is the complement of the closure of $\operatorname{Orb}(h, x)$. Then for every nonnegative integer $k$, $h^{-[k]}(G) \subset G$.

Proof. Let $k$ be a nonnegative integer and suppose that $h^{-[k]}(G)$ intersects the closure of $\operatorname{Orb}(h, x)$. Then because $h^{-[k]}(G)$ is open, it must intersect $\operatorname{Orb}(h, x)$; that is, there is a nonnegative integer $m$ such that $h^{[m]}(x)$ belongs to $h^{-[k]}(G)$. We conclude that $h^{[k+m]}(x)$ is in $G$, a contradiction. Thus $h^{-[k]}(G)$ does not intersect the closure of $\operatorname{Orb}(h, x)$, or $h^{-[k]}(G) \subset G$.

Theorem 2.3 (Separation Theorem). Suppose that $x \in X$ is such that $\operatorname{Orb}(f, x)$ is dense in $X$. The following are equivalent:

(a) $\operatorname{Orb}\left(f^{[2]}, x\right)$ is not dense in $X$;

(b) There exists a separation $\mathcal{D}_{1}, \mathcal{D}_{2}$ of $\mathcal{D}$ such that each of the sets $\mathcal{D}_{1}$ and $\mathcal{D}_{2}$ is invariant under $f^{[2]}$.

Proof. That (b) implies (a) is easy. Because $x$ is in $\mathcal{D}$, it is either in $\mathcal{D}_{1}$ or in $\mathcal{D}_{2}$. By the invariance of these sets under $f^{[2]}, \operatorname{Orb}\left(f^{[2]}, x\right)$ is contained entirely in $\mathcal{D}_{1}$ or entirely in $\mathcal{D}_{2}$ and thus can't be dense in $X$.

Now suppose that $\operatorname{Orb}\left(f^{[2]}, x\right)$ is not dense in $X$. Let $G$ be the complement of the closure of $\operatorname{Orb}\left(f^{[2]}, x\right)$ so that $G$ is nonempty and open. By Lemma 2.2 (with $\left.h=f^{[2]}\right)$, for each nonnegative integer $k, f^{-[2 k]}(G)$ is contained in $G$.

We claim that $f^{-[1]}(G)$ must be contained in the complement of $G$. To see this, suppose that $f^{-[1]}(G)$ intersects $G$. Because $G \cap f^{-[1]}(G)$ is open and $\operatorname{Orb}(f, x)$ is dense, there is a nonnegative integer $j$ such that $f^{[j]}(x)$ belongs to $G \cap f^{-[1]}(G)$. Observe that $j$ cannot be even $\left(f^{[j]}(x) \in G\right)$ and that $j$ cannot be odd $\left(f^{[j]}(x) \in\right.$ $f^{-[1]}(G)$ implies $f^{[j+1]}(x) \in G$ and $j+1$ is even), a contradiction. Thus $f^{-[1]}(G)$ must be contained in the complement of $G$.

Let $S_{1}=G$ and $S_{2}=f^{-[1]}(G)$. Note $S_{1}$ and $S_{2}$ are open ( $f$ is continuous) and disjoint. Let $w$ be in $\mathcal{D}$. Because $G$ is open and $w$ has dense orbit under $f$, there is a nonnegative integer $m$ such that $f^{[m]}(w) \in G$. Thus $w$ belongs to 
$f^{-[m]}(G)$ and is either in $S_{1}$ (if $m$ is even) or $S_{2}$ (if $m=2 k+1$ is odd, because then $\left.f(w) \in f^{-[2 k]}(G) \subset G\right)$; we have $\mathcal{D} \subset S_{1} \cup S_{2}$. Because $\mathcal{D}$ is dense, both $S_{1} \cap \mathcal{D}$ and $S_{2} \cap \mathcal{D}$ are nonempty. Thus the pair $\mathcal{D}_{1}:=S_{1} \cap \mathcal{D}$ and $\mathcal{D}_{2}:=S_{2} \cap \mathcal{D}$ is a separation of $\mathcal{D}$.

We complete the proof by showing $f\left(\mathcal{D}_{2}\right) \subset \mathcal{D}_{1}$ and $f\left(\mathcal{D}_{1}\right) \subset \mathcal{D}_{2}$. Suppose that $t \in \mathcal{D}_{2}$. Then because $t \in S_{2}, f(t)$ belongs to $G=S_{1}$. Because $f(t)$ also belongs to $\mathcal{D}$ (Lemma 2.1), $f(t) \in S_{1} \cap \mathcal{D}=\mathcal{D}_{1}$. Now, let $v \in \mathcal{D}_{1}$ so that, in particular, $v$ is in $G=S_{1}$. Suppose $f(v)$ also belongs to $G$; then $G \cap f^{-[1]}(G)$ is not empty (containing, e.g., $v$ ), but this contradicts $S_{1} \cap S_{2}=\emptyset$. Thus $f(v)$ is contained in the complement of $S_{1}=G$. In particular, $f(v)$ cannot be in $\mathcal{D}_{1}$; however, $f(v)$ is in $\mathcal{D}$ so that $f(v)$ must belong to $\mathcal{D}_{2}$.

In the following section, we present some applications of the Separation Theorem.

\section{The Decomposition Theorem}

The following theorem describes the behavior of orbits of $f$ relative to the open sets $S_{1}$ and $S_{2}$ that yielded the separation obtained in Theorem 2.3. The referee has pointed out to the author that for inverval maps this theorem is known.

Theorem 3.1 (Decomposition Theorem). Suppose that $x \in X$ is such that $\operatorname{Orb}(f, x)$ is dense in $X$, while $\operatorname{Orb}\left(f^{[2]}, x\right)$ is not dense. Let $S_{1}$ be the complement of the closure of $\operatorname{Orb}\left(f^{[2]}, x\right)$, and let $S_{2}=f^{-[1]}\left(S_{1}\right)$. Then

(a) $S_{1} \cap S_{2}=\emptyset$ and $S_{1} \cup S_{2}$ is dense in $X$;

(b) $f\left(S_{2}\right) \subset S_{1}$;

(c) $f\left(S_{1}\right) \subset X \backslash S_{1}$;

(d) $X \backslash\left(S_{1} \cup S_{2}\right)$ is invariant under $f$.

Proof. Part (b) follows immediately from the definition of $S_{2}$. Parts (a) and (c) are corollaries of the proof of the Separation Theorem. The second paragraph of that proof shows that $S_{2}$ is contained in the complement of $S_{1}$, while the fourth shows $S_{1} \cup S_{2}$ contains the dense set $\mathcal{D}$ and hence is dense itself. The last paragraph of the proof shows that (c) holds (the hypothesis that $v$ belong to $\mathcal{D}$ wasn't used to show that $f(v)$ belongs the closure of $\left.\operatorname{Orb}\left(f^{[2]}, x\right)\right)$.

Now we establish part (d). Let $w \in X \backslash\left(S_{1} \cup S_{2}\right)$. If $f(w)$ were in $S_{1}$, then $w$ would belong to $f^{-[1]}\left(S_{1}\right)=S_{2}$. If $f(w)$ were in $S_{2}$, then $f^{[2]}(w)$ would belong to $S_{1}$, and thus by Lemma 2.2, w would belong to $S_{1}$. Hence, $f(w)$ must belong to $X \backslash\left(S_{1} \cup S_{2}\right)$.

Given that the hypotheses of the preceding theorem hold, we see that an orbit that begins in $S_{1}$ either steps to $S_{2}$ and returns, or steps into $X \backslash\left(S_{1} \cup S_{2}\right)$ and remains there. An orbit that starts in $S_{2}$ steps into $S_{1}$ and either returns or steps into the invariant set $X \backslash\left(S_{1} \cup S_{2}\right)$. Observe that neither $S_{1}$ nor $S_{2}$ may contain a point having odd period under $f$. Also observe that the closed set $X \backslash\left(S_{1} \cup S_{2}\right)$ has no interior $\left(S_{1} \cup S_{2}\right.$ is dense).

Corollary 3.2. Suppose that $x \in X$ is such that $\operatorname{Orb}(f, x)$ is dense in $X$ and that the closure of the set of points of $X$ having odd period under $f$ has nonempty interior. Then $\operatorname{Orb}\left(f^{[2]}, x\right)$ is dense in $X$.

Proof. If $\operatorname{Orb}\left(f^{[2]}, x\right)$ were not dense in $X$, then the set of points having odd period under $f$ would be confined to the set $X \backslash\left(S_{1} \cup S_{2}\right)$, a closed set with no interior. 
We say that $f$ acts chaotically on $X$ provided that $f$ has an orbit dense in $X$ and that the set of periodic points of $f$ is dense in $X$ (by [2] this definition is equivalent to that given by Devaney in [4]).

Corollary 3.3. Suppose that $f$ acts chaotically on $X$ and that the closure of the set of points of $X$ having odd period under $f$ has nonempty interior. Then $f \circ f$ acts chaotically on $X$.

Proof. Since any point periodic for $f$ is periodic for $f \circ f$, the set of periodic points of $f \circ f$ is dense. Because $f$ acts chaotically on $X$, there exists $x \in X$ such that $\operatorname{Orb}(f, x)$ is dense in $X$. By Corollary 3.2, $\operatorname{Orb}\left(f^{[2]}, x\right)$ is also dense in $X$ so that $f \circ f$ has a dense orbit. Thus we see $f \circ f$ is chaotic, having the same sets of periodic points and points with dense orbit as does $f$.

Our final result is the one that inspired this paper: Ansari's Theorem in the $n=2$ case.

Theorem 3.4. Suppose that $B$ is a complex Banach space and $T: B \rightarrow B$ is bounded and linear. If for some $b \in B, \operatorname{Orb}(T, b)$ is dense in $B$, then $\operatorname{Orb}\left(T^{2}, b\right)$ is also dense in $B$.

Proof. If $\operatorname{Orb}(T, b)$ is dense in $B$, then the set $E=\{p(T) b: p$ is a polynomial $\} \backslash\{0\}$ is a dense set of vectors in $B$, each element of which has dense orbit ([3]). Because $E$ is connected and dense, the set $\mathcal{D}$ of vectors in $B$ having dense orbit under $T$ cannot be separated. Thus by the Separation Theorem, $\operatorname{Orb}\left(T^{2}, b\right)$ must be dense in $B$.

That linear maps may have dense orbits was first observed by Rolewicz [6]. That linear maps may satisfy, say, Devaney's definition of chaos was first observed by Shapiro and Godefroy [5].

The following example illustrates the Decomposition Theorem.

Example. Let $C_{1}$ be the unit circle in the complex plane $\mathbf{C}$, and let $L: \mathbf{C} \rightarrow \mathbf{C}$ be given by $L(z)=2 z-1$. Let $C_{2}=L\left(C_{1}\right)$ so that $C_{2}$ is the circle of radius 2 with center -1 and that $C_{1}$ is internally tangent to $C_{2}$ at 1 . Set $X=C_{1} \cup C_{2}$ and topologize $X$ using the euclidean metric. Define $g: X \rightarrow X$ by

$$
g(z)= \begin{cases}L\left(z^{2}\right) & \text { if } z \in C_{1}, \\ \left(L^{-1}(z)\right)^{2} & \text { if } z \in C_{2} .\end{cases}
$$

Verifying that $g$ is transitive is easy; thus, by the Baire Category Theorem, there is a point $x \in X$ having dense orbit under $g$. However, $g^{[2]}$ leaves both $C_{1}$ and $C_{2}$ invariant and thus has no dense orbits; in particular, $\operatorname{Orb}\left(g^{[2]}, x\right)$ is not dense in $X$. If $x$ is, for instance, in $C_{1}$, then the reader may verify that $S_{1}=C_{2} \backslash\{1\}$ and that $S_{2}=C_{1} \backslash\{-1,1\}$. Observe that $X \backslash\left(S_{1} \cup S_{2}\right)=\{-1,1\}$ is indeed invariant under $g$. We remark that the map $g$ is chaotic (any $\left(4^{n}-1\right.$ )-th root of unity is periodic for $g$ and the image under $L$ of any such root of unity is also periodic); hence, $g$ is an example of a chaotic map whose second iterate is not chaotic.

We conclude this paper with the following natural question:

For $n>2$, what kind of decomposition of $X$ may be obtained if one assumes that $f$ is (topologically) transitive on $X$ while $f^{[n]}$ is not? 


\section{ACKNOWLEDGMENT}

I wish to thank Joel Shapiro for bringing Ansari's work ([1]) to my attention and for his valuable criticism of a preliminary version of this paper. I would also like to thank the referee for providing several helpful suggestions.

\section{REFERENCES}

1. S. I. Ansari, Hypercyclic and cyclic vectors, J. Funct. Anal. 128 (1995), 374-383.

2. J. Banks, J. Brooks, G. Cairns, G. Davis, P. Stacey, On Devaney's Definition of Chaos, American Mathematical Monthly 99 (1992), 332-334. MR 93d:54059

3. P. S. Bourdon, Invariant manifolds of hypercyclic vectors, Proc. Amer. Math. Soc. 118 (1993), 845-847. MR 93i: 47002

4. R. L. Devaney, An introduction to chaotic dynamical systems, Addison-Wesley, Reading, MA, 1989. MR 91a:58114

5. G. Godefroy and J. H. Shapiro, Operators with dense, invariant, cyclic vector manifolds, J. Funct. Anal. 98 (1991), 229-269. MR 92d:47029

6. S. Rolewicz, On the orbits of elements, Studia Math. 32 (1969), 17-22. MR 39:3292

Department of Mathematics, Washington and Lee University, Lexington, Virginia 24450

E-mail address: pbourdon@wlu.edu 